\title{
Generation of Nonclassical Light by Unsaturated Two-Photon Absorption
}

\author{
Goggi Madhavi Latha, Meenakshisundaram Sripriya, Narayanan Ramesh \\ Department of Physics, A. M. Jain College, Meenambakkam, India \\ Email: nramesh1162@gmail.com
}

How to cite this paper: Latha, G.M., Sripriya, M. and Ramesh, N. (2017) Generation of Nonclassical Light by Unsaturated Two-Photon Absorption. Optics and Photonics Journal, 7, 139-150. https://doi.org/10.4236/opj.2017.79014

Received: August 15, 2017

Accepted: September 19, 2017

Published: September 22, 2017

Copyright @ 2017 by authors and Scientific Research Publishing Inc. This work is licensed under the Creative Commons Attribution International License (CC BY 4.0).

http://creativecommons.org/licenses/by/4.0/

(c) (i) Open Access

\begin{abstract}
In this paper, we investigate the distribution statistics of photons in a single mode radiation field subjected to two-photon absorption (TPA) and the factors that contribute to squeezing and antibunching of photons, leading to the generation of nonclassical light. TPA is a nonlinear optical phenomenon in which the atoms interact with the light field by absorbing two photons simultaneously. The motivation to study TPA is the recent intense activity on nanocrystallites/quantum dots. Further, it is the only nonlinear optical phenomenon that can be analytically studied. The simultaneous occurrence of squeezing and antibunching is studied with small initial photon numbers by solving the master equation for TPA of a single mode radiation directly by numerical integration, without going through analytical procedure. The results are compared with those of analytical/numerical procedures available. Further, the discussion on the parameters of squeezing and antibunching for short-time (ST) as well as long-time is done comprehensively in the present work by taking up the ST approximation and summation of ST (SST) procedure along with the exact numerical method.
\end{abstract}

\section{Keywords}

Two-Photon Absorption, Master Equation, Squeezing, Antibunching, Nonclassical Light

\section{Introduction}

A consequence of the quantization of radiation is the fluctuations associated with the zero point energy called vacuum fluctuations [1]. These fluctuations have no classical analog and are responsible for the generation of nonclassical light such as squeezed light and antibunched light. Another example of nonclassical light is the sub-Poisson light, which gives rise to a photon counting distri- 
bution narrower than Poisson distribution. The major interest in nonclassical light is that the noise is reduced below the standard quantum limit.

The coherent states of light exhibit minimum quantum noise and affect the quadratures, for example, amplitude and phase, equally. The correlations that can be introduced between them may reduce the noise in amplitude at the cost of increasing it in the phase. This is known as amplitude squeezed light [2] [3], wherein the photon number uncertainty $\left\langle(\Delta n)^{2}\right\rangle$ is reduced below $\langle n\rangle$, with a minimum limited by $\langle n\rangle^{1 / 3}$. This type of squeezing is observed with the interaction Hamiltonian of the form $a^{\dagger 2} a^{2}+$ h.c.

Another type of squeezed light involves reducing the fluctuations in one of the two standard orthogonal quadratures in such a way that the variance in that quadrature becomes less than the quantum noise limit of $1 / 4$. This is known as quadrature or ordinary squeezed light. The interaction Hamiltonian in this case is of the form $a^{\dagger 2}+$ h.c.

The intensity fluctuations of the optical field are described by the second-order correlation function [4]. This correlation function shows that a field whose photon probability distribution function narrower than Poissonian (sub-Poisson statistics) will have photons antibunched over certain time scale. This represents another type of nonclassical light called antibunched light. In general, squeezing, antibunching and sub-Poisson nature of light need not accompany one another.

To generate nonclassical light, normally, a coherent light is allowed to interact in a nonlinear fashion with a medium. The phase space contour line which is observed as a circle for the initial coherent state would then become an ellipse. A review of experiments, main achievements and progress made in technology used in the production and detection of quadrature squeezed light, from the first successful production in 1985 to 2015 is presented in [5]. An evaluation of suitability of third-order susceptibility materials, especially semiconductors, as squeezers is discussed in [6].

Although squeezing and antibunching of photons are observed in light generated by a variety of nonlinear optical processes, it is useful to study the process that admits analytical solution and one such process is two-photon absorption (TPA). Moreover, TPA attracts further interest as it may allow squeezing and antibunching to occur simultaneously.

Normally, the master equation for TPA involving the reduced density matrix operator of the single mode light field is solved by the generating function method [7] [8] [9]. Then one obtains the factorial moments of photon number in order to verify the nonclassical nature of light. Though the generating function approach is an analytical procedure, in the last stage this requires numerical computation. It is also of interest to study TPA in a short time soon after the interaction of light with matter begins [10]. The master equation for TPA was solved directly by Garcia-Fernandez et al. [11] using the eigenvalue method, without going through any analytical procedure, for initial photon numbers 1, 10, 20, 30 and 60 and a comparative study was made with short-time approximation. An 
explicit analytical expression for squeezing parameter resulting from a strong coherent beam is obtained [12] and shown how squeezing can be controlled by varying detuning parameter, involving both dispersion (proportional to the real part of $\chi^{(3)}$ ) and absorption (proportional to the imaginary part of $\chi^{(3)}$ ) coefficients. A study [13] on the evolution of nonclassical states of light from TPA medium for two different cases of initial states, a squeezed coherent state and an eigenstates of the two photon annihilation operator (even and odd coherent states) is made on the fluctuations in photon number operator and in the quadrature components of the field.

The study on photon statistics of the internal and the external fields of a microcavity [14] containing a TPA medium using Langevin equation approach shows a strong photon number squeezing for the output field, whereas the internal field shows a weak squeezing. This study was made with the assumption of a large initial photon number and a very small one-photon absorption rate. It is reported [15] that a thin film of CdSe nanocrystals embedded in PMMA exposed to laser radiation showed a strong TPA over the bulk at $800 \mathrm{~nm}$, occurring due to the confinement of system and the study demonstrated the importance of reduction of scattering losses in a nonlinear medium used for the generation of squeezed light. Recently, a study of TPA in nanocrystallites [16], organic molecules [17] and quantum dots [18] has attracted attention of many researchers. Ginossar et al. [19] suggested a process to study the rate at which correlations among polarization entangled photons of two-mode squeezed vacuum transferred to the electrons in a semiconductor, enabling them to crossover from positive to negative spin correlation, even with large number of photons.

In this paper, we consider Hamiltonian of the form $a^{\dagger 2}+$ h.c. for the generation of quadrature or ordinary squeezed light using TPA. An attempt has been made to solve the master equation for TPA of a single mode radiation field numerically without going through analytical procedure and obtain the required factorial moments as a function of the dimensionless time parameter for different initial photon numbers. In $\$ 2$, the theory behind the photon statistics is developed by defining the parameters required to describe antibunching and squeezing of photons. To discuss the parameters of that describe squeezing and antibunching for short-time (ST) as well as long-time comprehensively, we take up the ST approximation, summation of ST (SST) procedure and exact numerical integration method. $\$ 3$ gives the methods of solving the master equation. Finally, the results are discussed in $\$ 4$.

\section{Theoretical Background}

\subsection{Antibunching, Sub-Poisson Light and Squeezing}

The intensity fluctuations of the optical field are described by the correlation function $G^{(2)}$. The degree of second order coherence is a measure of the correlation of the light intensities at two space-time points. It is defined in terms of the positive and negative frequency parts of the light field. 
For a single mode radiation field, the normalized form of $G^{(2)}$ in terms of the creation $a^{\dagger}$ and annihilation $a$ operators of the field is given by

$$
g^{(2)}=\left\langle a^{\dagger} a^{\dagger} a a\right\rangle /\left\langle a^{\dagger} a\right\rangle^{2}
$$

or

$$
g^{(2)}-1=Q /\left\langle a^{\dagger} a\right\rangle
$$

where

$$
Q=\left(\left\langle\left(a^{\dagger} a\right)^{2}\right\rangle-\left\langle a^{\dagger} a\right\rangle^{2}-\left\langle a^{\dagger} a\right\rangle\right) /\left\langle a^{\dagger} a\right\rangle
$$

is called the Mandel parameter. In terms of number operator $n=a^{\dagger} a$, we have $\left\langle a^{\dagger} a\right\rangle=\langle n\rangle$ and variance $\left\langle(\Delta n)^{2}\right\rangle=\left\langle n^{2}\right\rangle-\langle n\rangle^{2}$. For Poisson statistics, $\left\langle(\Delta n)^{2}\right\rangle=\langle n\rangle$ and hence $g^{(2)}=1(Q=0)$. This is true for coherent light where the uncertainties in the two quadratures are equal and photons are randomly distributed. If $g^{(2)}>1(Q>0)$ then the photon number fluctuation follows super-Poisson statistics and photons are bunched. Finally, if $g^{(2)}<1(Q<0)$ sub-Poisson statistics is obeyed and this corresponds to antibunched light where photons lose correlations. Thus the sign of $Q$ becomes an important factor for the confirmation of both sub-Poissonian and antibunching in a single mode light field.

Squeezing exists in the quadrature $X_{1}$ or $X_{2}$ of the light, defined by

$$
X_{1}=(1 / 2)\left(a+a^{\dagger}\right)
$$

and

$$
X_{2}=(1 / 2 i)\left(a-a^{\dagger}\right)
$$

if the uncertainty in $X_{1}$ or $X_{2}$ described by the parameter $S_{1,2}<0$. i.e.

$$
S_{1,2}=\left\langle X_{1,2}^{2}\right\rangle-\left\langle X_{1,2}\right\rangle^{2}-1 / 4<0
$$

or

$$
S_{1,2}=(1 / 2)\left(\left\langle a^{\dagger} a\right\rangle-\left\langle a^{\dagger}\right\rangle\langle a\rangle \pm \operatorname{Re}\left(\left\langle a^{\dagger 2}\right\rangle-\left\langle a^{\dagger}\right\rangle^{2}\right)\right)<0 .
$$

The maximum squeezing achievable by any process corresponds to $S_{1}=-0.25$ and for antibunched (sub-Poisson) light the minimum value of Mandel parameter $Q$ is -1 . For TPA, with initial coherent light $\alpha=|\alpha| \mathrm{e}^{i \varphi}$, where photon number is $\left|\alpha^{2}\right|$, in the stationary or steady state [20], i.e. in the limit the dimensionless time parameter $\tau \rightarrow \infty$, it is found that $\left\langle a^{\dagger} a\right\rangle \sim 1 / 2,\left\langle a^{\dagger}\right\rangle=\alpha^{*} /|\alpha| \sqrt{2 \pi}$ and $\left\langle a^{\dagger 2}\right\rangle=0$, for $|\alpha|^{2} \gg 1$, implying $S_{1}(\infty)=0.091$. Therefore, in the stationary state there is no squeezing, whereas, $Q(\infty)=-1 / 2$, minimum value for TPA, indicating antibunching and hence the presence of sub-Poisson light.

\subsection{Master Equation for Two-Photon Absorption}

In TPA process, the atoms and the light field interact by the simultaneous absorption of two photons. It is assumed here that almost all the atoms are main- 
tained in the ground state and hence two-photon emission can be ignored. During this interaction, the statistical properties of light field change and they depend on the initial conditions of the incident light.

We assume that the single mode radiation field of frequency $\omega$ interacts with an ensemble of $N$ two-level atoms with a transition frequency of $\omega_{0}$, resonantly via TPA $\left(2 \omega=\omega_{0}\right)$. A relatively small part of them are assumed to be excited during interaction and hence we call it the unsaturated TPA [8] [9].

The total Hamiltonian $H$, describing the interaction of electromagnetic field with a nonlinear medium is

$$
H=H_{F}+H_{A}+H_{I},
$$

where $H_{F}$ is the Hamiltonian operator of the field, $H_{A}$ Hamiltonian operator of atoms and $H_{I}$ is the interaction Hamiltonian. Therefore,

$$
H=\hbar \omega a^{\dagger} a+\frac{1}{2} \hbar \omega_{0} \sum_{i}\left(c_{2 i}^{\dagger} c_{2 i}-c_{1 i}^{\dagger} c_{1 i}\right)+\hbar \sum_{i}\left(K c_{2 i}^{\dagger} c_{1 i} a a+K^{*} c_{1 i}^{\dagger} c_{2 i} a^{\dagger} a^{\dagger}\right) .
$$

Here $c$ and $c^{\dagger}$ operators refer to the ground state and excited state of the $i^{\text {th }}$ atom. The equation of motion for the density operator in terms of the Hamiltonian is

$$
i \hbar \mathrm{d} \rho / \mathrm{d} t=[H, \rho] .
$$

The above Liouville equation is a particular case of a generalized equation under Markovian approximation [21]. The generalized Liouville equation

$$
\mathrm{d} \rho / \mathrm{d} t=\mathcal{L}[\rho],
$$

where $\mathcal{L}$ is a linear operator that generates a finite super operator called Lindblad operator. This operator includes all possible quantum jumps during the interaction of the field with the medium.

Retaining only the relevant TPA absorption and emission terms, the equation of motion for the density operator of the light field [7] [8] is

$$
\mathrm{d} \rho / \mathrm{d} t=k_{1}\left\{\left[a a \rho, a^{\dagger} a^{\dagger}\right]+\left[a a, \rho a^{\dagger} a^{\dagger}\right]\right\}+k_{2}\left\{\left[a^{\dagger} a^{\dagger} \rho, a a\right]+\left[a^{\dagger} a^{\dagger}, \rho a a\right]\right\},
$$

where the two terms on right hand side represent the absorption and emission processes of TPA. As mentioned in the beginning of this section, neglecting the emission term the master equation becomes

$$
\mathrm{d} \rho / \mathrm{d} \tau=-\left(a^{\dagger 2} a^{2} \rho-2 a^{2} \rho a^{\dagger 2}+\rho a^{\dagger 2} a^{2}\right),
$$

where $\tau=2 k_{1} t$ is the dimensionless time parameter. $k_{1}$ depends on the line shape function and intensity of the radiation field and also on the number density of atoms of the medium. It is related to the TPA absorption coefficient $\beta$ as

$$
k_{1}=n_{0} \hbar \omega c^{2} \beta / 8 V,
$$

where

$$
\beta=3 \omega \chi_{I}^{(3)} / 2 n_{0}^{2} \varepsilon_{0} c^{2} .
$$


$n_{0}$ is the refractive index and $\chi_{I}^{(3)}$ is imaginary part of third-order susceptibility of the medium for TPA. The diagonal and off-diagonal matrix elements of the density operator in the Fock representation are given by $\rho_{n, m}=\langle n|\rho| m\rangle$ and they satisfy

$$
\mathrm{d} \rho_{n, n} / \mathrm{d} \tau=(n+1)(n+2) \rho_{n+2, n+2}-n(n-1) \rho_{n, n}
$$

and

$$
\begin{aligned}
\mathrm{d} \rho_{n, m} / \mathrm{d} \tau= & {[(n+1)(n+2)(m+1)(m+2)]^{1 / 2} \rho_{n+2, m+2} } \\
& -(1 / 2) n(n-1) \rho_{n, m}-(1 / 2) m(m-1) \rho_{n, m}
\end{aligned}
$$

where, $m=n+\mu$, here $\mu$ denotes the degree of off-diagonality. For an initial coherent light, the diagonal elements of density matrix $(\mu=0)$ can be written as

$$
\rho_{n, n}(0)=\langle n \mid \alpha\rangle\langle\alpha \mid n\rangle=\exp \left(-\left|\alpha^{2}\right|\right)|\alpha|^{2 n} / n ! .
$$

The off-diagonal elements of density matrix $(\mu \neq 0)$ are

$$
\rho_{n, n+\mu}(0)=\langle n \mid \alpha\rangle\langle\alpha \mid n+\mu\rangle=\exp (-i \mu \varphi) \exp \left(-\left|\alpha^{2}\right|\right) \alpha^{n}\left(\alpha^{*}\right)^{n+\mu} / \sqrt{n !(n+\mu) !} .
$$

Following [22], to avoid square root of factorials in off-diagonal elements, we define

$$
\rho_{n, n+\mu}(\tau)=\sqrt{n ! /(n+\mu) !} \Psi_{n}(\mu, \tau)
$$

$$
\begin{aligned}
& \text { At } \tau=0 \text {, } \\
& \Psi_{n}(\mu, 0)=\sqrt{(n+\mu) ! / n !} \rho_{n, n+\mu}(0)=\exp (-i \mu \varphi) \exp \left(-\left|\alpha^{2}\right|\right)|\alpha|^{2 n}\left(\alpha^{*}\right)^{\mu} / n ! .
\end{aligned}
$$

The master equation can be rewritten in terms of $\Psi_{n}$ as

$$
\mathrm{d} \Psi_{n}(\mu, \tau) / \mathrm{d} \tau=\left[n(n-1)+n \mu+\frac{1}{2} \mu(\mu-1)\right] \Psi_{n}(\mu, \tau)+(n+1)(n+2) \Psi_{n+2}(\mu, \tau) .
$$

The relevant expectation values for the evaluation of antibunching and squeezing are given by

$$
\begin{gathered}
\left\langle a^{\dagger} a\right\rangle=\sum_{n} n \Psi_{n}(0, \tau), \\
\left\langle a^{\dagger 2} a^{2}\right\rangle=\sum_{n} n(n-1) \Psi_{n}(0, \tau), \\
\left\langle\left(a^{\dagger} a\right)^{2}\right\rangle=\sum_{n} n^{2} \Psi_{n}(0, \tau), \\
\left\langle a^{\dagger}\right\rangle=\sum_{n} \Psi_{n}(1, \tau), \\
\left\langle a^{\dagger 2}\right\rangle=\sum_{n} \Psi_{n}(2, \tau) .
\end{gathered}
$$

\section{Evaluation of Antibunching and Squeezing}

\subsection{Generating Function Method}

Usually, the master equation is solved exactly by using a generating function that describes the change of photon statistics of an initially coherent light and $\Psi_{n}(\mu, \tau)$ is determined by the nth order derivative of the generating function. 
This leads to an expression involving an infinite sum over gamma functions with complex arguments, along with an exponential factor $\exp [-n(n-1) \tau]$. This factor helps in convergence of the infinite sum, allowing truncation of the series at suitable $n$ value. Further evaluation of relevant moments is done by numerical methods [8] [9] [22] [23].

\subsection{Short-Time (ST) Approximation}

It is of interest to check if any change in photon statistics occurs soon after the interaction of light with matter and also to avoid the labor involved in obtaining the exact solution describe above, short-time expansion procedure [10] is followed. The expectation values for shorter time intervals $(\tau \ll 1)$ are

$$
\begin{gathered}
\left\langle a^{\dagger} a\right\rangle=|\alpha|^{2}-2|\alpha|^{4} \tau+2|\alpha|^{4}\left(2|\alpha|^{2}+1\right) \tau^{2}, \\
\left\langle a^{\dagger}\right\rangle=|\alpha| \mathrm{e}^{-i \varphi}\left[1-|\alpha|^{2} \tau+(1 / 2)|\alpha|^{2}\left(3|\alpha|^{2}+1\right) \tau^{2}\right], \\
\left\langle a^{\dagger 2}\right\rangle=|\alpha|^{2} \mathrm{e}^{-2 i \varphi}\left[1-\left(2|\alpha|^{2}+1\right) \tau+\left(4|\alpha|^{4}+4|\alpha|^{2}+1 / 2\right) \tau^{2}\right], \\
\left\langle a^{\dagger 2} a^{2}\right\rangle=|\alpha|^{4}-2|\alpha|^{4}\left(2|\alpha|^{2}+1\right) \tau+3|\alpha|^{4}\left(4|\alpha|^{4}+6|\alpha|^{2}+1\right) \tau^{2} .
\end{gathered}
$$

\subsection{Summation of Short-Time Expansion (SST)}

For studying long time behavior of photon statistics, a summation of all higher order terms is necessary [20]. This imposes a restriction on the convergence limit of series on the quantity $\xi=2|\alpha|^{2} \tau<1$, for greater initial photon number $|\alpha|^{2} \gg 1$. The convergence domain limit $\xi<1$ can be enlarged by analytical continuation to study the long time behaviour of photon statistics but with small initial photon number as well. The expectation values obtained for longer time intervals are

$$
\begin{gathered}
\left\langle a^{\dagger} a\right\rangle=|\alpha|^{2} /(1+\xi)+\xi^{2}(3+\xi) / 6(1+\xi)^{3}, \\
\left\langle a^{\dagger}\right\rangle=\alpha^{*} /(1+\xi)^{1 / 2}+\xi^{2} \alpha^{*} / 8|\alpha|^{2}(1+\xi)^{5 / 2}, \\
\left\langle a^{\dagger 2}\right\rangle=\exp (-2 i \varphi) \exp (-\tau)\left\langle a^{\dagger} a\right\rangle, \\
\left\langle a^{\dagger 2} a^{2}\right\rangle=|\alpha|^{4} /(1+\xi)^{2}-|\alpha|^{2} \xi /(1+\xi)^{4} .
\end{gathered}
$$

\subsection{Numerical Solution of Master Equation}

The master equation was solved directly using the eigenvalue method [11], without going through any analytical procedure. It was solved exactly by Laplace transformation procedure for the diagonal elements of k-photon absorption [24].

In the present work, $\Psi_{n}(\mu, \tau)$ is obtained directly by the numerical integration of the master Equation (22) by employing a standard integration procedure [25] with adaptive step-size control, using a personal computer with a program 
written in FORTRAN 95 language. The numerical technique involves a series of estimates of $\Psi_{n}(\mu, \tau)$ by changing step-size to achieve good accuracy by monitoring the first moment, for different values of $n$ at various $\tau$-values, starting from the coherent state of light given by Equation (21), with initial photon numbers $|\alpha|^{2}=1$ and 9. This procedure is followed for $\mu=0,1,2$ to estimate the diagonal and off-diagonal elements of $\Psi_{n}(\mu, \tau)$ and are substituted in the expressions for the expectation values given by Equations (23) to (27) to determine antibunching parameter $Q$ and squeezing $S_{1}$, using Equations ((3) and (7)) respectively.

\section{Results and Discussion}

The results and discussion on the parameters of squeezing and antibunching for short-time (ST) as well as long-time is done in the present work comprehensively for comparison purpose by taking up the ST approximation, summation of ST (SST) procedure and exact numerical method. To check the reliability of our numerical results, a comparison of the variation of diagonal elements $\rho_{n, n}$ as a function of $n$ for various dimensionless time $\tau$ values, for the initial photon number 9 is made with those of Simaan and Loudon [22] and found to agree very well.

From Figure 1, which represents $S_{1}$ as a function of dimensionless time parameter $\tau$ for $|\alpha|=1$, determined by the ST (a), SST (b) methods along with our numerical result (c), it is observed that up to $\tau=0.06$ all three curves coincide, after which ST value starts deviating, reaches a minimum at 0.11 and thereafter makes a transition toward zero value. On the other hand, the SST values agree pretty well with the exact numerical value till a minimum is reached. Compared to $\mathrm{ST}$, the minimum of $S_{1}$, corresponding to the maximum squeezing, achieved is more by a factor of 2.3 approximately and the squeezing persists for a longer duration. Further, it is noticed that $S_{1}$ does not take positive values for large

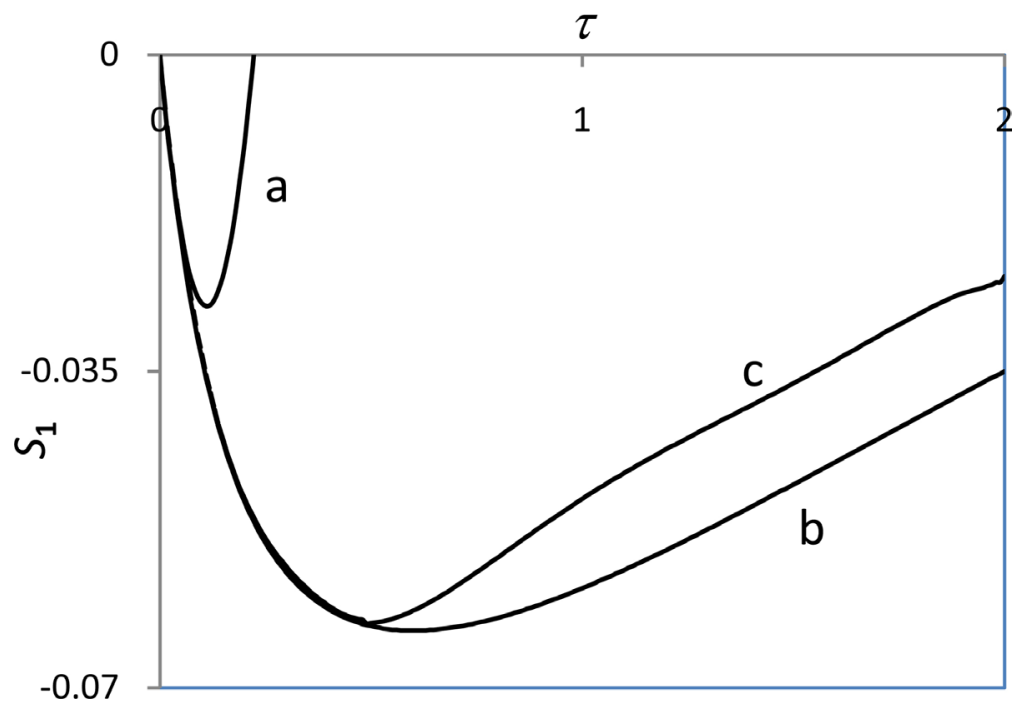

Figure 1. Variation of $S_{1}$ with $\tau$ for $|\alpha|=1$, (a) ST; (b) SST; and (c) Our result. 


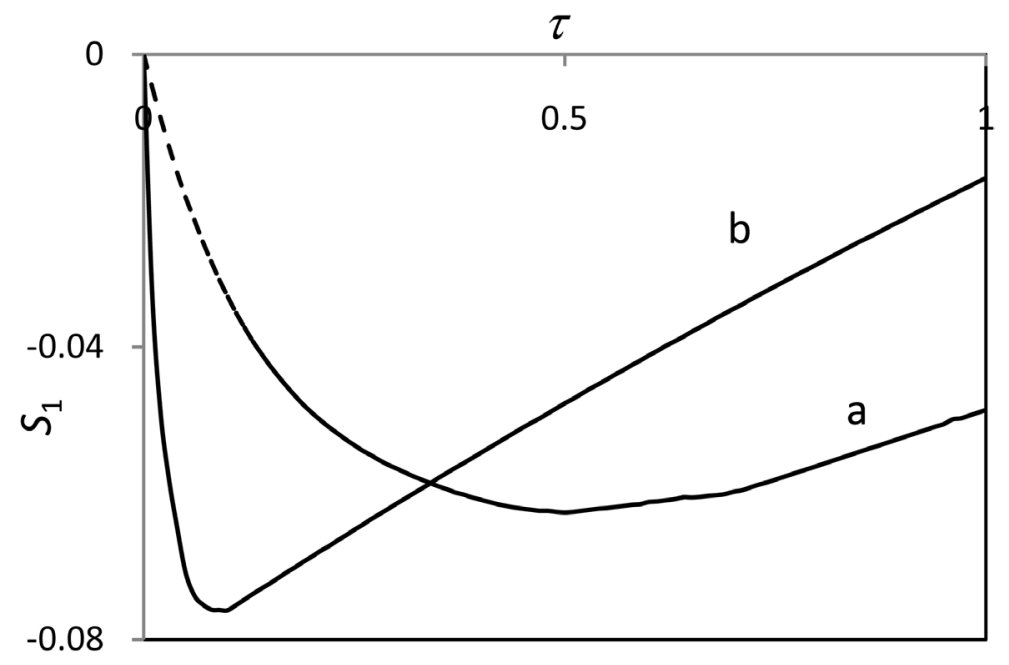

Figure 2. Variation of $S_{1}$ with $\tau$ for (a) $|\alpha|=1$ and (b) $|\alpha|=3$.

$\tau$-values, in fact it moves close to zero only, as indicated by Garcia-Fernandez et al. [11] and Loudon [10]. The above observations are also true for $|\alpha|=3$, except that squeezing is lost at large times, becomes zero at $\tau=1.4$ and reaches the stationary value of 0.091 at about 7.0. So, it appears that with regard to evaluation of the amount of maximum squeezing and the time at which it is attained, it is only suffice to use the expression of SST and avoid the complicated exact numerical procedure.

In Figure 2, we present the variation of $S_{1}$, obtained from our numerical procedure, as a function of $\tau$ for $|\alpha|=1$ and 3 as well. As the initial photon number is increased, the minimum value of $S_{1}$ is deeper and moves toward shorter time, but the duration of squeezing is smaller. Our results agree very well with those of Agarwal and Hildred [9]. The maximum squeezing achieved by increasing the initial photon number to 9 is about $30 \%$ of the upper limit of -0.25 . By increasing the initial number further, the maximum squeezing that could be attained in TPA is $33 \%$ or $1 / 3$ rd of the minimum limit only. It corresponds to $1.8 \mathrm{~dB}$ noise reduction in the quadrature $X_{1}$. In other words, maximum squeezing can be achieved much earlier with more initial photons, even for as low as 10 , but is lost much quickly. It is to be noted that this reduction in noise corresponds to the squeezing along the standard quadrature $X_{1}$, orthogonal to $X_{2}$. It is customary to represent the quadrature squeezing by introducing a pair of rotated quadratures $Y_{1}$ and $Y_{2}$ related to the standard quadratures $X_{1}$ and $X_{2}$ through the quadrature angle $\theta$. This is called squeeze angle as the squeezing is maximum along this direction. Thus, it is possible to have more squeezing along one of the rotated quadratures than that with the standard one [26]. This assertion concurs with the experimental result of optomechanical squeezing of light [27].

Finally, Figure 3 represents the behavior of the Mandel parameter $Q$ as a function of $\tau$ calculated from our numerical method for $|\alpha|=1$ and 3. It is observed that antibunching or anticorrelation of photons, as indicated by $Q<0$, 


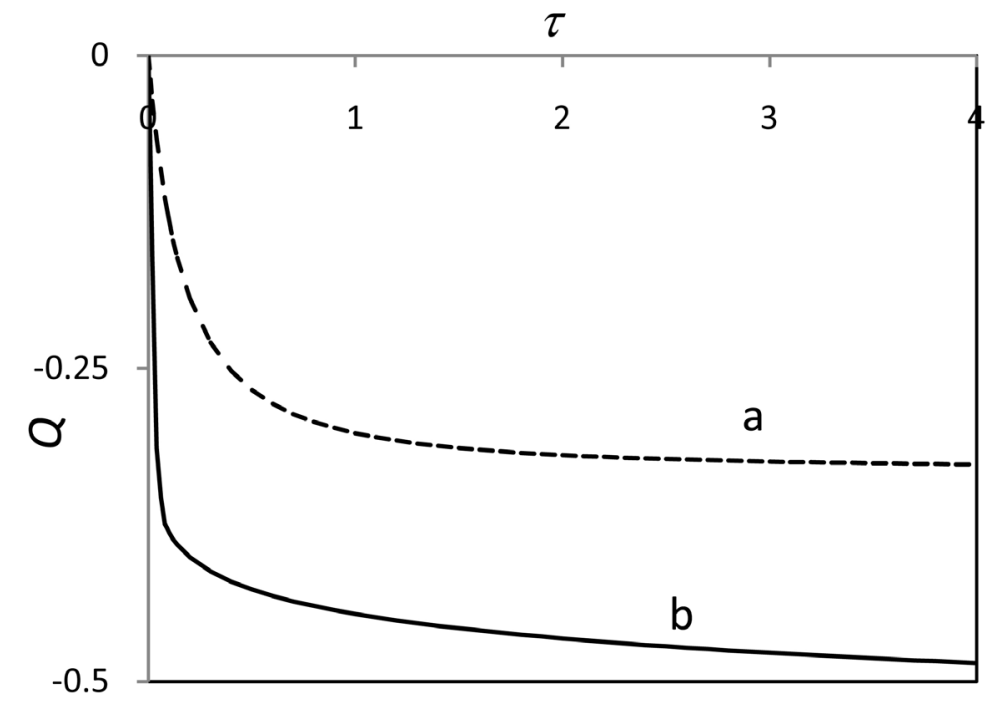

Figure 3. Variation of $Q$ with $\tau$ for (a) $|\alpha|=1$ and (b) $|\alpha|=3$.

takes place much earlier for large initial photon number. The analysis of the results indicates that the minimum value of $Q$ that is achievable in TPA is $-1 / 2$, the stationary state value itself, even at a time of about 7.0 and it remains constant thereafter, with fewer than 10 initial photons. In other words, for initial photon number $|\alpha|^{2}>9$ the maximum antibunching corresponding to the stationary state value is reached much sooner, whereas, SST calculation of Mandel parameter predicts only a minimum value of $-1 / 3$ against $-1 / 2$.

Summarizing, after the interaction of initially coherent light with a two-photon absorber, it becomes nonclassical by acquiring squeezing and antibunching of photons. Though squeezing is lost after some time, the light remains an antibunched one.

\section{Conclusions}

The simultaneous observation of squeezing and antibunching is made with small initial photon numbers by solving the master equation for TPA of a single mode radiation directly by numerical integration, without going through analytical procedure. Further, the discussion on the parameters of squeezing and antibunching for short-time (ST) as well as long-time is done comprehensively in the present work by taking up the ST approximation, summation of ST (SST) procedure and exact numerical method.

The results obtained by us agree well with those of already existing analyti$\mathrm{cal} /$ numerical procedures in the literature. The nonclassical parameters of light calculated by the ST approximation deviate very much from those of SST and exact numerical methods, except for exceedingly small time intervals in the beginning of interaction.

It is observed from our analysis that to know the amount of maximum squeezing and the time at which it is attained, it is only sufficient to use the expression of SST and avoid the complicated exact numerical procedure. On increasing the 
initial photon number, the minimum value of squeezing parameter is deeper and moves toward shorter time, but the duration of squeezing is smaller. The maximum amount of squeezing that could be attained in TPA is $33 \%$ or $1 / 3^{\text {rd }}$ (corresponding to $1.8 \mathrm{~dB}$ noise reduction in the standard quadrature) of the lowest achievable value of -0.25 .

The Mandel parameter describing antibunching of photons obtained from the numerical method reaches the stationary state value of $-1 / 2$ even at earlier times for initial photon number $\geq 9$ and remains unchanged thereafter.

Thus, after acquiring the nonclassical characteristics of squeezing and antibunching on interaction with a two-photon absorber, the initial coherent light loses squeezing after some time but antibunching of photons persists.

\section{References}

[1] Orszag, M. (2000) Quantum Optics. Springer, Berlin. https://doi.org/10.1007/978-3-662-04114-7

[2] Kitagawa, M. and Yamamoto, Y. (1986) Number-Phase Minimum-Uncertainty State with Reduced Number Uncertainty in a Kerr Nonlinear Interferometer. Physical Review A, 34, 3974-3988. https://doi.org/10.1103/PhysRevA.34.3974

[3] Drummond, P.D. and Walls, D.F. (1980) Quantum Theory of Optical Bistability. I. Nonlinear Polarisability Model. Journal of Physics A: Mathematical and General, 13, 725-741. https://doi.org/10.1088/0305-4470/13/2/034

[4] Loudon, R. and Knight, P.L. (1987) Squeezed Light. Journal of Modern Optics, 34, 709-759. https://doi.org/10.1080/09500348714550721

[5] Anderson, U.L., Gehring, T., Marquardt, C. and Leuchs, G. (2016) 30 Years of Squeezed Light Generation. Physica Scripta, 91, Article ID: 053001. https://doi.org/10.1088/0031-8949/91/5/053001

[6] Hilico, J., Courty, M., Fabre, C., Giacobino, E., Abram, I. and Oudar, J.L. (1992) Squeezing with $\chi^{(3)}$ Materials. Applied Physics B, 55, 202-209. https://doi.org/10.1007/BF00325007

[7] Agarwal, G.S. (1970) Field-Correlation Effects in Multiphoton Absorption Processes. Physical Review A, 1, 1445-1459. https://doi.org/10.1103/PhysRevA.1.1445

[8] Tornau, N. and Bach, A. (1974) Quantum Statistics of Two-Photon Absorption. Optics Communications, 11, 46-49 https://doi.org/10.1016/0030-4018(74)90330-7

[9] Agarwal, G.S. and Hildred, G.P. (1986) Time Development of Squeezing in Two Photon Absorption. Optics Communications, 58, 287-289. https://doi.org/10.1016/0030-4018(86)90452-9

[10] Loudon, R. (1984) Squeezing in Two-Photon Absorption. Optics Communications, 49, 67-70. https://doi.org/10.1016/0030-4018(84)90092-0

[11] Garcia-Fernandez, P., De Los Terreros, L.S. and Bermejo-Barrera, F.J. (1986) Squeezing Parameters in Two-Photon Absorption. Optica Acta, 33, 945-948. https://doi.org/10.1080/713822050

[12] Agarwal, G.S. (1987) Squeezing in Two Photon Absorption from a Strong Coherent Beam. Optics Communications, 62, 190-192. https://doi.org/10.1016/0030-4018(87)90025-3

[13] Gilles, L. and Knight, P.L. (1993) Two-Photon Absorption and Nonclassical States of Light. Physical Review A, 48, 1582-1593. https://doi.org/10.1103/PhysRevA.48.1582 
[14] Ezaki, H. (1999) Photon Number Squeezing Due to Two-Photon Absorption in Internal and External Fields of a Microcavity. Journal of the Society of Japan, 68 , $1562-1566$.

[15] Ispasoiu, R.G., Jin, Y., Lee, J., Papadimitrakopoulos, F. and Goodson, R. (2002) Two-Photon Absorption and Photon-Number Squeezing with CdSe Nanocrystals. Nano Letters, 2, 127-130. https://doi.org/10.1103/PhysRevA.48.1582

[16] Ebothe, J., Kityk, I.V. and Fuks-Janczarek, I. (2006) Two-Photon Absorption Study of the Large-Sized Nanocrystallites. Applied Surface Science, 252, 5763-5767. https://doi.org/10.1016/j.apsusc.2005.07.055

[17] Li, W., Feng, J., Ren, A., Zhang, X. and Sun, C. (2009) Theoretical Investigation on the One- and Two-photon Absorption Properties of Porphyrin-Thiophene Chromophores. Chinese Journal of Chemistry, 27, 1269-1279. https://doi.org/10.1002/cjoc.200990212

[18] More, D., Rajesh, Ch., Lad, A.D., Ravindra Kumar, G. and Mahamuni, S. (2010) Two Photon Absorption in $\mathrm{Mn}^{2+}$-Doped ZnSe Quantum Dots. Optics Communications, 283, 2150-2154. https://doi.org/10.1016/j.optcom.2010.01.053

[19] Ginossar, E., Levinson, Y. and Levit, S. (2008) Optical Manipulation of Collective Spin Correlations in Semiconductors with a Squeezed Vacuum of Polarized Photons. Physical Review B, 77, Article ID: 035307. https://doi.org/10.1103/PhysRevB.77.035307

[20] Bandilla, A. (1987) Simple Analytical Formulae for Squeezing in Unsaturated Two-Photon Absorption. Journal of Modern Optics, 34, 903-907.

https://doi.org/10.1080/09500348714550821

[21] Nuclear Science and Engineering. https://ocw.mit.edu/courses/nuclear-engineering/

[22] Simaan, H.D. and Loudon, R. (1978) Off-Diagonal Density Matrix for Single-Beam Two-Photon Absorbed Light. Journal of Physics A: Mathematical and General A, 11, 435-441. https://doi.org/10.1088/0305-4470/11/2/018

[23] Gerry, C.C. and Hach III, E.E. (1993) Enhanced Squeezing from a Kerr Medium Combined with Two-Photon Absorption. Optics Communications, 100, 211-214. https://doi.org/10.1016/0030-4018(93)90582-P

[24] Voigt, H., Bandilla, A. and Ritze, H.H. (1980) Exact Analytical Solution for the Change of the Photon Statistics Due to k-Photon Absorption. Zeitschrift fur Physik B Condensed Matter and Quanta, 36, 295-302. https://doi.org/10.1007/BF01325293

[25] Press, W.H., Teukolsky, S.A., Vellarling, W.T. and Flannery, B.P. (1992) Numerical Recipes in FORTRAN. 2nd Edition, Cambridge University Press, New Delhi.

[26] Gerry, C.G. and Knight, P.L. (2005) Introductory Quantum Optics. Cambridge University Press, Cambridge.

[27] Purdy, T.P., Yu, P.L., Peterson, R.W., Kampel, N.S. and Regal, C.A. (2013) Strong Optomechanical Squeezing of Light. Physical Review X, 3, Article ID: 031012. https://doi.org/10.1103/PhysRevX.3.031012 
Submit or recommend next manuscript to SCIRP and we will provide best service for you:

Accepting pre-submission inquiries through Email, Facebook, LinkedIn, Twitter, etc. A wide selection of journals (inclusive of 9 subjects, more than 200 journals)

Providing 24-hour high-quality service

User-friendly online submission system

Fair and swift peer-review system

Efficient typesetting and proofreading procedure

Display of the result of downloads and visits, as well as the number of cited articles Maximum dissemination of your research work

Submit your manuscript at: http://papersubmission.scirp.org/

Or contactopj@scirp.org 